\title{
A CONSTRUÇÃO TEÓRICO-METODOLÓGICA DA ABORDAGEM CONSTRUCIONISTA, CONTEXTUALIZADA E SIGNIFICATIVA
}

\author{
Danielle Aparecida do Nascimento dos Santos ${ }^{1}$, Elisa Tomoe Moriya Schlünzen ${ }^{2}$ \\ ${ }^{1}$ Universidade do Oeste Paulista - UNOESTE, curso de Pedagogia, Presidente Prudente, SP.. ${ }^{2}$ Universidade Estadual \\ Paulista - UNESP, Programa de Pós-Graduação em Educação, Presidente Prudente, SP. E-mail: danielle@unoeste.br
}

\section{RESUMO}

O atual paradigma da escola inclusiva implica em mudanças substanciais em toda a dinâmica dos sistemas de ensino, especialmente no fazer pedagógico. As práticas pedagógicas, em uma perspectiva inclusiva, só podem ocorrer mediante a abertura incondicional às diferenças, ao desenvolvimento de saberes, à reflexão sobre a ação, estruturando sua prática a partir de situações significativas, trabalhando com temas e projetos. Quando o ensino passa a ser concebido a partir do contexto e vivência dos estudantes, surgem as metodologias ativas, o trabalho com projetos, a incorporação e impregnação de Tecnologias Digitais de Informação e Comunicação (TDIC) e a valorização das diferenças. Esses princípios são as bases da abordagem Construcionista, Contextualizada e Significativa (CCS), vivenciada em pesquisas vinculadas ao Grupo de Pesquisa Ambientes Potencializadores para a Inclusão (API). Neste artigo será apresentado o constructo teórico-metodológico que estruturou essa abordagem de ensino denominada CCS.

Palavras-chave: Abordagem Construcionista, Contextualizada e Significativa, Educação Inclusiva, Formação de Professores.

\section{A THEORETICAL- METHODOLOGICAL CONSTRUCTION OF THE CONSTRUCTIONIST, CONTEXTUALIZED AND SIGNIFICANT APPROACH}

\begin{abstract}
The current paradigm of inclusive school implies substantial changes in the whole dynamic of education systems, especially in pedagogical practice. Pedagogical practices in an inclusive perspective, can only occur through unconditional openness to differences, the development of knowledge, to reflect on the action, structuring your practice from significant situations, working with themes and designs. When teaching becomes designed from the context and experience of students, come the active methodologies, project work, embedding and impregnation Digital Technologies of Information and Communication and appreciation of differences. These principles are the basis of constructivist approach, contextual and meaningful, experienced in research linked to enhancers Environments Research Group for Inclusion. This paper deals with the theoretical and methodological construct that this structured teaching approach called CCS.
\end{abstract}

Keywords: Constructionist approach, Contextual and Meaningful, Inclusive Education, Teacher Training. 


\section{INTRODUÇÃO}

As abordagens de ensino são definidas a partir de estudos sobre como se configura e se constrói o conhecimento humano que tem, em sua origem e desenvolvimento, referenciais e estímulos que determinam a sua configuração. Pode-se afirmar, diante disso, que o motivo que condiciona a construção do conhecimento humano é elemento que faz parte do seu contexto, a exemplo da cultura, da sociedade e da educação.

Em educação, a relação entre o ensino e a aprendizagem é fonte de pesquisas ao longo da história, desde que a didática passou a ser problematizada, assim como as formas com que os seres humanos incorporam os estímulos vivenciados gerando aprendizado. O ensino é um ato intencional que pode ou não ter as suas consequências na aprendizagem planejadas, a priori.

Mizukami (1986) esclarece que, algumas abordagens de ensino apresentam claro referencial filosófico e psicológico, enquanto outras são mais intuitivas ou fundamentadas na própria prática ou na imitação de modelos. Com isso, as consequências da ação educativa, a partir das abordagens, sempre têm um referencial teórico implícito ou explícito que vai fundamentar a visão de mundo, dos sujeitos, da aprendizagem, de cultura, de sociedade e de conhecimento.

Esse referencial teórico que caracteriza a ação educativa são as abordagens, tendências ou linhas pedagógicas, ou seja, o referencial que sustenta essa visão e que pode e deve oferecer diretrizes para a ação docente.

De acordo com Mizukami (1986), cinco abordagens foram se configurando ao longo da história da educação brasileira: tradicional, comportamentalista, humanista, sociocultural e cognitivista. No eixo de pesquisa do uso de Tecnologias Digitais de Informação e Comunicação (TDIC) em educação, foram definidas duas abordagens de ensino que podem ser relacionadas com as abordagens caracterizadas pela autora. As abordagens instrucionista e construcionista (PAPERT, 1986).

Ao vivenciar a tese Mudanças nas Práticas Pedagógicas do Professor: Criando um Ambiente Construcionista, Contextualizado e Significativo para Crianças com Necessidades Especiais Físicas, Schlünzen (2000) estabeleceu meios para novas práticas pedagógicas de professores da Associação de Assistência à Criança Deficiente (AACD), usando como estratégia o desenvolvimento de projetos para criar um ambiente Construcionista, Contextualizado e Significativo (CCS), favorecendo o processo de ensino e de aprendizagem das crianças com deficiências. 0 computador ou a tecnologia foram utilizados como recursos potencializadores para a aprendizagem dos conceitos disciplinares. 
A vivência do ambiente CCS revelou que: os estudantes, dentro de um projeto contextualizado, cujo tema foi escolhido por eles mesmos, puderam vivenciar, pensar, criar, expressar e aprender; os estudantes passaram a ser mais participativos, sem exceção, porque puderam aprender brincando, de maneira significativa, já que o assunto fez parte do contexto que estavam vivenciando; os conceitos das diferentes disciplinas foram formalizados ao mesmo tempo, sem ter que abordá-los separadamente e, principalmente, foram relacionados com a vivência dos estudantes; as relações se modificaram, tornaram-se mais harmoniosas e menos baseadas em estereótipos ou pré-conceitos criados em função das características físicas ou intelectuais de cada um; ao desenvolver projetos, criaram-se possibilidades do professor intervir e auxiliar os estudantes a compreender o que estavam fazendo e, com isso, assimilar os conceitos. Sem essa intervenção do professor, o projeto poderia ficar apenas no "fazer" e não no "compreender"; o foco da pesquisa foi trabalhar com os professores para criar um ambiente CCS no cotidiano da sala de aula para crianças com deficiências.

De acordo com os resultados do processo de construção do ambiente CCS, afirmou-se na referida tese que a vivência e os resultados permitiram vislumbrar perspectivas de transformação para a melhoria dos processos de ensino e aprendizagem dos estudantes. Esses e outros aspectos presentes no desenvolvimento do ambiente CCS permitiram, naquele momento, desenvolver uma abordagem metodológica CCS. De acordo com Schlünzen (2000) nessa abordagem é possível articular os conceitos com o currículo, contextualizando-os e atribuindo significado a eles.

A abordagem CCS foi desenvolvida ao longo da trajetória de pesquisa, ensino e extensão do Grupo de Pesquisa Ambientes Potencializadores para a Inclusão (API). Por isso, faz-se necessário e importante desvelar o constructo teórico metodológico e princípios da abordagem CCS.

\section{METODOLOGIA}

Considerando que os conhecimentos construídos a partir do objeto de estudo (a abordagem CCS) em si representa uma análise teórico-metodológica do próprio conceito de abordagem e do conceito de ensino, a metodologia para a construção do presente artigo é de natureza qualitativa, uma vez que, para a construção da referida abordagem houve uma análise de ambientes naturais como fontes diretas de dados e o contato direto dos pesquisadores com o ambiente e as situações a serem investigadas (LÜDKE; ANDRÉ, 1986).

Além disso, como o processo de elaboração, execução e análise do constructo teóricometodológico da abordagem permitiu que os pesquisadores realizassem inferências ao longo do mesmo, por isso optamos por considerar a pesquisa como do tipo investigação-ação, uma vez que 
foi proposta uma ação reflexiva e dialógica empreendida no trabalho para o coletivo (FRANCO, 2005).

A partir desses elementos que caracterizam a abordagem da pesquisa, seguem os resultados e discussão a respeito do conceito de abordagem CCS.

\section{RESULTADOS}

Retomando as definições de abordagens de ensino delimitadas por Mizukami (1986), na abordagem tradicional, de caráter liberalista, a ação educativa é caracterizada pela transmissão de informações que a humanidade acumulou ao longo dos tempos. O professor, detentor dessas informações, executa uma prática transmissiva e o estudante, em um papel de aluno, as recebe. 0 objeto do ensino, que é o conteúdo, é meramente "depositado" nos sujeitos e, além disso, os conteúdos não fazem parte da realidade ou cotidiano dos estudantes.

A abordagem comportamentalista tem bases sociológicas, uma vez que compreende o ensino como determinante para moldar o comportamento social dos indivíduos. Por isso, os objetivos de ensino são estabelecidos a partir do condicionamento que podem inferir nos estudantes. De acordo com Libâneo (2004), essa abordagem tem ênfase na tecnologia educacional, no sentido tecnicista, e o conceito da abordagem está diretamente ligado às máquinas de ensinar de Skinner, que fazem referência à abordagem instrucionista de ensino, em que, de acordo com Schlünzen (2000), o computador exerce a função de quem transmite a informação por meio de um programa, ou seja, ele cumpre um papel semelhante ao de um professor que passa as informações.

No caso da abordagem humanista, ao invés do enfoque ser dado ao conteúdo ou às ferramentas de ensino, é voltado ao sujeito. O ensino passa a ser centrado no estudante e o objetivo é o crescimento do indivíduo e a organização pessoal da realidade, a fim de que a pessoa se desenvolva em múltiplos aspectos. O professor, diferente da abordagem tradicionalista, que é um mero transmissor de informações, ou na abordagem comportamentalista, que é somente um organizador de conteúdos, passa a ser um "facilitador da aprendizagem", devendo fornecer meios, estímulos e instrumentos para que o estudante aprenda. De acordo com Saviani (1984), essa abordagem é caracterizada como pedagogia nova e a primeira com tendência não diretiva e antiautoritária de ensino.

Outra abordagem definida por Mizukami (1986) é a sociocultural, que tem origem no trabalho de Freire (1970) e na alfabetização de adultos. A abordagem considera que a ação educativa não deve ser restrita à educação formal e que a educação é um ato político, que 
proporciona ao indivíduo uma visão crítica do mundo e da realidade que ele pode transformar e melhorar. Bordenave (1984) define essa abordagem como a "pedagogia da problematização".

Finalmente, Mizukami (1986) define a abordagem cognitivista, em que a organização do conhecimento é elemento central do processo de ensino. Conhecida também como abordagem piagetiana, seu enfoque se dá no caráter interacionista entre sujeito e objeto, e a aprendizagem é decorrente da assimilação e da modificação das estruturas mentais já existentes.

Bordenave (1984) também relacionada a abordagem cognitivista à pedagogia da problematização, uma vez que seu foco está na valorização do processo de transformação dos sujeitos, enquanto agentes transformadores de sua própria realidade. Nesse sentido, o estudante é um sujeito ativo e transformador, o que gera um "entusiasmo construtivo", ou seja, uma motivação para a descoberta e a aprendizagem.

No caso das abordagens de ensino relacionadas ao uso de TDIC em educação, a abordagem instrucionista tem suas bases na abordagem comportamentalista, e a abordagem construcionista, tem bases que podem ser observadas nas abordagens humanista e cognitivista.

De acordo com Schlünzen (2000), na abordagem construcionista a tecnologia é usada para o aprendiz resolver problemas, por meio da formalização, explicitação e construção do conhecimento. Nessa abordagem, o estudante exerce o papel de quem usa a tecnologia, por intermédio de um software, para explicitar suas ideias, ao invés de ser ensinado por um computador, como ocorre na abordagem instrucionista. Assim, o estudante é motivado a produzir algo palpável.

Dentro dessa proposta, os trabalhos pioneiros de Papert (1985) e Valente (1991) destacamse com a implementação e utilização da linguagem de programação Logo. Ainda de acordo com Schlünzen (2000), a abordagem construcionista consiste em criar situações-problema que permitam ao estudante resolver questões reais e aprender com o uso e com a experiência dos conceitos envolvidos em suas resoluções.

A concepção da abordagem CCS é fundamentada em três teorias: piagetiana, vigotskiana e freireana. A teoria piagetiana traz importantes vertentes que contribuem para a abordagem construcionista, uma vez que seu enfoque é interacionista e cognitivista, baseia-se na ideia de que a pessoa vai descobrindo o sentido do mundo por meio da ação (que é ponto central do funcionamento intelectual) sobre pessoas e sobre os objetos.

Para Piaget (1974), o processo de construção cognitiva se constitui a partir de uma evolução das estruturas de pensamento que permitem a apropriação do conhecimento. Nesse sentido, a aprendizagem seria quase que uma consequência do desenvolvimento cognitivo. $\mathrm{Na}$ 
abordagem CCS, essa construção dá-se de maneira que o uso de tecnologias altera as estruturas do pensamento (mentais), por meio da interação do estudante com os mais diversos recursos, proporcionando uma reflexão constante com o papel de depurar os processos de constituição de tal interação.

As bases vigotskianas passam a contribuir nesse processo, uma vez que Vygotsky (1989) concebe o aprendizado como um resultado do desenvolvimento mental. Para tanto, estabelece dois níveis de desenvolvimento: o desenvolvimento real, das funções mentais que já se estabeleceram como resultado de certos ciclos de desenvolvimento já completados; e o desenvolvimento potencial, que o indivíduo consegue realizar com a ajuda de outros.

Dessa maneira, a ação comunicativa e a manipulação de objetos, em um ambiente que valorize a cooperação para a construção de algo que expresse as diferentes habilidades, permite avanços nos níveis de desenvolvimento dos indivíduos.

Contudo, para que realmente o sujeito seja capaz de desenvolver suas potencialidades, é necessária uma mudança real nas práticas pedagógicas, que podem ser potencializadas por meio do trabalho com projetos ou outras metodologias ativas, permitindo que se crie uma ponte entre a sociedade e o estudante, conforme evidenciado na pesquisa de Santos (2007), considerando que o interpsicológico influencia no intrapsicológico.

Encontramos na teoria freireana uma prática pedagógica que vise à educação libertária de seus educandos, demonstrando que são capazes de agir diante de uma situação concreta. Com isso, na abordagem CCS a prática pedagógica deve estar além da simples instrução, fazendo com que a prática deixe de ser "bancária", ou seja, fazendo com que o professor deixe de "depositar" informações nos estudantes.

Em um contexto em que são criados mecanismos para operacionalizar a abordagem CCS, seja na formação inicial e continuada de professores, seja no contexto da escola, seja em contextos polissêmicos de ensino e aprendizagem, as TDIC são utilizadas para auxiliar na construção do conhecimento dos estudantes e possibilitar que encontrem a sua autoimagem, para poderem atuar como seres pensantes em nossa sociedade.

Dessa forma, à medida que o estudante ou aprendiz vê seu interesse individual ser transformado num contexto social e/ou científico, verá suas habilidades afloradas e o processo de ensino será enriquecido. 


\section{DISCUSSÃO}

As experiências de 15 anos vivenciadas no grupo de pesquisa API, configurada a partir de iniciativas de inclusão digital, escolar e social de Estudantes Público Alvo da Educação Especial (pessoas com deficiências, transtornos globais do desenvolvimento e altas habilidades ou superdotação) por meio da formação de professores, demonstram que a reorganização do currículo mediante o trabalho com projetos em uma abordagem denominada CCS (SCHLÜNZEN, 2005) tem permitido que as TDIC sejam utilizadas como recursos para uma aprendizagem mais autodirigida e inclusiva, mediante as perspectivas teóricas supracitadas.

Os principais resultados desse constructo teórico-metodológico podem ser observados nas pesquisas de pós-graduação descritas no Quadro 1, que tem fazem menção em seu título ou objetivos diretamente à abordagem CCS:

Quadro 1. Síntese das Pesquisas de Mestrado/Doutorado sobre a Abordagem CCS

\begin{tabular}{|l|l|l|}
\hline Título da Pesquisa & Nível & $\begin{array}{l}\text { Ano } \\
\text { Conclusão }\end{array}$ \\
\hline $\begin{array}{l}\text { A Formação de Professores de uma Escola da Rede Pública } \\
\text { Estadual em Serviço para o Trabalho com Projetos } \\
\text { Utilizando as Tecnologias de Informação e Comunicação }\end{array}$ & Mestrado & 2007 \\
\hline $\begin{array}{l}\text { A Abordagem Construcionista, Contextualizada e } \\
\text { Significativa na Formação de Professores para uma escola } \\
\text { Inclusiva }\end{array}$ & Doutorado & 2014 \\
\hline $\begin{array}{l}\text { Construção da Abordagem CCS no Trabalho Colaborativo } \\
\text { entre os Professores de Matemática e o Especializado em } \\
\text { Deficiência Intelectual }\end{array}$ & Mestrado & Em \\
\hline $\begin{array}{l}\text { Ações Pedagógicas Inovadoras no Ensino Superior: } \\
\text { Inserção da Modalidade Semipresencial e as Abordagens } \\
\text { CCS e EJV }\end{array}$ & Doutorado & Em \\
\hline $\begin{array}{l}\text { O Designer Educacional na construção de cursos em EaD } \\
\text { segundo a abordagens CCS e o EJV }\end{array}$ & Doutorado & Em \\
\hline $\begin{array}{l}\text { Abordagem Construcionista, Contextualizada } \\
\text { e Significativa: Formação, Extensão e Pesquisa em uma } \\
\text { perspectiva inclusiva }\end{array}$ & Livre-Docência & 2015 \\
\hline
\end{tabular}

Cumpre ressaltar que as pesquisas descritas no Quadro 1 destacam-se entre as 17 pesquisas de mestrado concluídas, 3 pesquisas de mestrado em andamento, 1 pesquisa de doutorado concluída e 5 pesquisas de doutorado em andamento, vinculadas ao Grupo API. 


\section{CONCLUSÃO}

A abordagem CCS indica que é fundamental o professor estar disposto a modificar sua prática, gerando um constante processo de formação e reflexão na ação, utilizando projetos e as TDIC, ou seja, ele tem que perceber a importância de um novo papel a que se propõe. Seu papel na abordagem CCS ultrapassa os limites da transmissão de informações, chegando no mais íntimo do ser de um estudante que, como qualquer outra pessoa, merece ser formado para aflorar seu caráter, personalidade, competência e habilidades.

A abordagem CCS proporciona o desenvolvimento da reflexão e elaboração crítica sobre as ações, dentro de projetos reais e motivadores, partindo dos interesses individuais e coletivos. Assim, essa abordagem pode tomar forma a partir de mudanças na concepção da formação de professores, do uso de TDIC, do contexto e vivência dos estudantes e do paradigma inclusivista.

\section{REFERÊNCIAS}

BORDENAVE, J. E. D. A opção pedagógica pode ter consequências individuais e sociais importantes. In: Revista da Educação AEC, n. 54, 1984.

FRANCO, M. A. S. A pedagogia da pesquisa-ação. Educação e Pesquisa. Revista da Faculdade de Educação da USP. Vol. 31, fascículo 3, p. 483-502. dez 2005.

FREIRE, P. Pedagogia do oprimido. São Paulo: Paz e Terra, 1970.

LIBÂNEO, J. C. Didática. São Paulo: Cortez, 2004. p. 221-247.

LUDKE, M.; ANDRÉ, M. Pesquisa em educação: abordagens qualitativas. São Paulo: EPU, 1986.

MIZUKAMI, M. G. N. Ensino: as abordagens do processo. São Paulo: Pedagógica e Universitária, 1986.

PAPERT, S. A New Opportunity for Elementary Science Education. Cambridge, Massachusetts: Massachusetts Institute of Technology, 1986.

PIAGET, J. Aprendizagem e conhecimento. Trad. Equipe Livraria Freitas Barros. Rio de Janeiro: Freitas Barros, 1974.

SANTOS, D. A. N. A formação de professores de uma escola da rede pública estadual em serviço para o trabalho com projetos usando as Tecnologias de Informação em Comunicação. 2007.

Dissertação (Mestrado em Educação) - Faculdade de Ciências e Tecnologia, Universidade Estadual Paulista "Júlio de Mesquita Filho", Presidente Prudente, 2007.

SAVIANI, D. Escola e democracia. São Paulo: Cortez, 1984. 
SCHLÜNZEN, E. T. M. A tecnologia para inclusão de pessoas com necessidades especiais (PNE). In: PELLANDA, N. M. C.; SCHLÜNZEN, E. T. M.; SCHLÜNZEN JUNIOR, K. (Org.). Inclusão digital: tecendo redes afetivas/cognitivas. Rio de Janeiro: DP\&A, 2005, p. 195-210. 1 v.

SCHLÜNZEN, E. T. M. Mudanças nas práticas pedagógicas do professor: criando um ambiente construcionista contextualizado e significativo para crianças com necessidades especiais físicas. 2000. 212 p. Tese (Doutorado em Educação: Currículo) - Pontifícia Universidade Católica de São Paulo, São Paulo, 2000.

VALENTE, J. A. Liberando a mente: computadores na educação especial. Campinas: Gráfica Central da Unicamp, 1991.

VYGOTSKY, L. S. Pensamento e linguagem. Tradução: Jefferson Luiz Camargo. São Paulo, Martins Fontes, 1989. 